\title{
Wide QRS Tachycardia by ECG Finding
}

National Cancer Institute

\section{Source}

National Cancer Institute. Wide QRS Tachycardia by ECG Finding. NCI Thesaurus. Code C71090.

An electrocardiographic finding of three or more consecutive wide QRS complexes of uncertain orig in with a rate greater than a certain threshold (100 or 120 beats per minute are commonly used). (CDISC) 